\title{
MACKEY'S CRITERION FOR SUBGROUP RESTRICTION OF KRONECKER PRODUCTS AND HARMONIC ANALYSIS ON CLIFFORD GROUPS
}

\author{
TULLIO CECCHERINI-SILBERSTEIN, FABIO SCARABOTTI, AND FILIPPO TOLLI
}

\begin{abstract}
We present a criterion for multiplicity-freeness of the decomposition of the restriction $\operatorname{Res}_{H}^{G}\left(\rho_{1} \otimes \rho_{2}\right)$ of the Kronecker product of two generic irreducible representations $\rho_{1}, \rho_{2}$ of a finite group $G$ with respect to a subgroup $H \leq G$. This constitutes a generalization of a well known criterion due to Mackey (which corresponds to the case $H=G)$. The corresponding harmonic analysis is illustated by detailed computations on the Clifford groups $G=\mathbb{C L}(n)$, together with the subgroups $H=\mathbb{C L}(n-1)$, for $n \geq 1$, which lead to an explicit decomposition of the restriction of Kronecker products.
\end{abstract}

\section{Contents}

1. Introduction

2. Preliminaries

3. Harmonic analysis for the pair $(G \times G \times H, \tilde{H})$

3.1. A Frobenius reciprocity type theorem and proof of the main result

3.2. Invariant vectors and spherical characters

4. Harmonic analysis on Clifford groups

4.1. Clifford groups and their irreducible representations

4.2. Kronecker products of irreducible representations of $\mathbb{C L}(n)$

4.3. The orbits of $\mathbb{C L}(n)$ on $\mathbb{C L}(n) \times \mathbb{C L}(n)$

4.4. The spherical characters for $(\mathbb{C L}(n) \times \mathbb{C L}(n) \times \mathbb{C L}(n), \widetilde{\mathbb{C L}(n)})$ References

\section{INTRODUCTION}

Let $G$ be a finite group and let $K \leq G$ be a subgroup.

We denote by $\widehat{G}$ a complete set of pairwise inequivalent irreducible representations of $G$ and say that a $G$-representation is multiplicity-free provided it decomposes as the direct sum of distinct elements in $\widehat{G}$. Let also denote by $L(G)$ the group algebra of $G$ and by ${ }^{K} L(G)^{K}=\left\{f \in L(G): f\left(k_{1} g k_{2}\right)=f(g) \forall g \in G, \forall k_{1}, k_{2} \in K\right\}$ the subalgebra of

Date: May 2, 2019.

1991 Mathematics Subject Classification. 20C15, 43A90, 20 G40.

Key words and phrases. Representation theory of finite groups, Gelfand pair, Mackey's criterion, Kronecker product, Clifford groups. 
bi- $K$-invariant functions on $G$. Recall that $(G, K)$ is a Gelfand pair provided ${ }^{K} L(G)^{K}$ is commutative.

Our main result is the following criterion for multiplicity-freeness of the restriction to $H$ of Kronecker products of irreducible representations of $G$ (this is a generalization of Mackey's criterion corresponding to the case $H=G$ ).

Theorem 1.1. Let $G$ be a finite group and $H \leq G$ a subgroup. Consider the subgroup $\widetilde{H}=\{(h, h, h): h \in H\}$ of $G \times G \times H$. Then the following conditions are equivalent:

(i) $(G \times G \times H, \widetilde{H})$ is a Gelfand pair;

(ii) $\operatorname{Res}_{H}^{G}\left(\rho_{1} \otimes \rho_{2}\right)$ is multiplicity-free for all $\rho_{1}, \rho_{2} \in \widehat{G}$.

We shall deduce this result from a suitable Frobenius' reciprocity type theorem for permutation representations (Theorem 3.1) which is quite interesting on its own, and illustate it by explicit computations on the Clifford groups $\mathbb{C L}(n)$ (see Section 4) yielding the following results.

Theorem 1.2. (i) $(\mathbb{C L}(n) \times \mathbb{C L}(n) \times \mathbb{C L}(n), \widetilde{\mathbb{C L}(n)})$ is a Gelfand pair;

(ii) $(\mathbb{C L}(n) \times \mathbb{C L}(n) \times \mathbb{C L}(n-1), \mathbb{C L}(n-1))$ is a Gelfand pair if and only if $n$ is odd.

In addition, we give a complete harmonic analysis for the Gelfand pair $(\mathbb{C L}(n) \times \mathbb{C L}(n) \times$ $\mathbb{C L}(n), \widetilde{\mathbb{C L}(n)})$ with an explicit description of the associated spherical characters (see Section 4.4) and of the orbits of $\mathbb{C L}(n)$ acting by conjugation on the Cartesian product $\mathbb{C L}(n) \times \mathbb{C L}(n)($ see Section 4.3$)$.

\section{Preliminaries}

In this section, in order to fix notation, we recall some basic facts on linear algebra and representation theory of finite groups.

All vector spaces considered here are complex. Moreover, we shall equip every finite dimensional vector space $V$ with a scalar product denoted by $\langle\cdot, \cdot\rangle_{V}$ with associated norm $\|\cdot\|_{V}$; we usually omit the subscript if the vector space we are referring to is clear from the context. Given two finite dimensional vector spaces $W$ and $U$, we denote by $\operatorname{Hom}(W, U)$ the vector space of all linear maps from $W$ to $U$, and for $T \in \operatorname{Hom}(W, U)$ we denote by $T^{*} \in \operatorname{Hom}(U, W)$ the adjoint of $T$. We define a (normalized Hilbert-Schmidt) scalar product on $\operatorname{Hom}(W, U)$ by setting

$$
\left\langle T_{1}, T_{2}\right\rangle_{\operatorname{Hom}(W, U)}=\frac{1}{\operatorname{dim} W} \operatorname{tr}\left(\mathrm{T}_{2}^{*} \mathrm{~T}_{1}\right)
$$

for all $T_{1}, T_{2} \in \operatorname{Hom}(W, U)$, where $\operatorname{tr}(\cdot)$ denotes the trace of linear operators; note that, by centrality of the trace (so that $\operatorname{tr}\left(\mathrm{T}_{2}^{*} \mathrm{~T}_{1}\right)=\operatorname{tr}\left(\mathrm{T}_{1} \mathrm{~T}_{2}^{*}\right)$ ), we have

$$
\left\langle T_{1}, T_{2}\right\rangle_{\operatorname{Hom}(W, U)}=\frac{\operatorname{dim} U}{\operatorname{dim} W}\left\langle T_{2}^{*}, T_{1}^{*}\right\rangle_{\operatorname{Hom}(U, W)}
$$

In particular, the map $T \mapsto \sqrt{\operatorname{dim} U / \operatorname{dim} W} T^{*}$ is an isometry from $\operatorname{Hom}(W, U)$ onto $\operatorname{Hom}(U, W)$. Finally, note that denoting by $I_{W}: W \rightarrow W$ the identity operator, we have $\left\|I_{W}\right\|_{\operatorname{Hom}(W, W)}=1$. 
Let $G$ be a finite group. A unitary representation of $G$ is a pair $(\sigma, W)$ where $W$ is a finite dimensional vector space and $\sigma: G \rightarrow \operatorname{Hom}(W, W)$ is a group homomorphism such that $\sigma(g)$ is unitary (that is, $\sigma(g)^{*} \sigma(g)=I_{W}$ ) for all $g \in G$. The term "unitary" will be omitted. We denote by $d_{\sigma}=\operatorname{dim}(W)$ the dimension of the representation $(\sigma, W)$. Let $(\sigma, W)$ be a $G$-representation. A subspace $V \leq W$ is said to be $G$-invariant provided $\sigma(g) V \subseteq V$ for all $g \in G$. Writing $\left.\sigma\right|_{V}(g)=\left.\sigma(g)\right|_{V}$ for all $g \in G$, we have that $\left(\left.\sigma\right|_{V}, V\right)$ is a $G$-representation, called a subrepresentation of $\sigma$. One says that $\sigma$ is irreducible provided the only $G$-invariant subspaces are trivial (equivalently, $\sigma$ admits no proper subrepresentations).

Let $(\sigma, W)$ and $(\rho, U)$ be two $G$-representations. We denote by $\operatorname{Hom}_{G}(W, U)=\{T \in$ $\operatorname{Hom}(W, U): T \sigma(g)=\rho(g) T, \forall g \in G\}$, the space of all intertwining operators. Observe that if $T \in \operatorname{Hom}_{G}(W, U)$ then $T^{*} \in \operatorname{Hom}_{G}(U, W)$. Indeed, for all $g \in G$ we have

$$
T^{*} \rho(g)=T^{*} \rho\left(g^{-1}\right)^{*}=\left(\rho\left(g^{-1}\right) T\right)^{*}=\left(T \sigma\left(g^{-1}\right)\right)^{*}=\sigma\left(g^{-1}\right)^{*} T^{*}=\sigma(g) T .
$$

One says that $(\sigma, W)$ and $(\rho, U)$ are equivalent, and we shall write $(\sigma, W) \sim(\rho, U)$ (or simply $\sigma \sim \rho$ ), if there exists a bijective intertwining operator $T \in \operatorname{Hom}_{G}(W, U)$.

We denote by $\widehat{G}$ a complete set of pairwise-inequivalent irreducible representations of $G$ (it is well known (cf. [2, Theorem 3.9.10]) that there is a bijection between $\widehat{G}$ and the set of conjugacy classes of elements in $G$ so that, in particular, $\widehat{G}$ is finite). Moreover, if $\sigma, \rho \in \widehat{G}$ we set $\delta_{\sigma, \rho}=1$ (resp. $\left.=0\right)$ if $\sigma \sim \rho$ (resp. otherwise).

Suppose that $H \leq G$ is a subgroup. We denote by $\left(\operatorname{Res}_{H}^{G} \sigma, W\right)$ the restriction of $\sigma$ to $H$, that is, the $H$-representation defined by $\left[\operatorname{Res}_{H}^{G} \sigma\right](h)=\sigma(h)$ for all $h \in H$.

The direct sum of the two representations $\sigma$ and $\rho$ is the representation $(\sigma \oplus \rho, W \oplus U)$ defined by $[(\sigma \oplus \rho)(g)](w, u)=(\sigma(g) w, \rho(g) u)$ for all $g \in G, w \in W$ and $u \in U$.

If $\sigma$ is a subrepresentation of $\rho$, then denoting by $W^{\perp}=\left\{u \in U:\langle u, w\rangle_{U}=0 \forall w \in W\right\}$ the orthogonal complement of $W$ in $U$, we have that $W^{\perp}$ is a $G$-invariant subspace and $\rho=\left.\sigma \oplus \rho\right|_{W^{\perp}}$. From this one deduces that every representation $\rho$ decomposes as a direct sum of irreducible subrepresentations. In the particular case when these irreducible subrepresentations are pairwise inequivalent, we say that $\rho$ is multiplicity-free.

More generally, when $\sigma$ is equivalent to a subrepresentation of $\rho$, we say that $\sigma$ is contained in $\rho$.

Suppose that $(\sigma, W)$ is irreducible. Then the number $m=\operatorname{dimHom}_{G}(W, U)$ denotes the multiplicity of $\sigma$ in $\rho$. This means that one may decompose $U=U_{1} \oplus U_{2} \oplus \cdots \oplus U_{m} \oplus U_{m+1}$ with $\left(\left.\rho\right|_{U_{i}}, U_{i}\right) \sim(\sigma, W)$ for all $i=1,2, \ldots, m$. The $G$-invariant subspace $U_{1} \oplus U_{2} \oplus \cdots \oplus$ $U_{m} \leq U$ is called the $W$-isotypic component of $U$ and is denoted by $m W$. One also says that $\rho$ (or, equivalently, $U$ ) contains $m$ copies of $\sigma$ (resp. of $W$ ). If this is the case, we say that $T_{1}, T_{2}, \ldots, T_{m} \in \operatorname{Hom}_{G}(W, U)$ yield an isometric orthogonal decomposition of $m W$ if $T_{i} \in \operatorname{Hom}_{G}\left(W, U_{i}\right)$ and, in addition,

$$
\left\langle T_{i} w_{1}, T_{j} w_{2}\right\rangle_{U}=\left\langle w_{1}, w_{2}\right\rangle_{W} \delta_{i, j}
$$

for all $w_{1}, w_{2} \in W$ and $i, j=1,2, \ldots, m$. This implies that the subrepresentation of $U$ isomorphic to $m W$ is equal to the orthogonal direct sum $T_{1} W \oplus T_{2} W \oplus \cdots \oplus T_{m} W=$ $U_{1} \oplus U_{2} \oplus \cdots \oplus U_{m}$ and each operator $T_{j}$ is a isometry from $W$ onto $U_{j}$. 
Lemma 2.1. Let $(\sigma, W)$ be an irreducible $G$-representation. Then the operators $T_{1}, T_{2}, \ldots, T_{m}$ yield an isometric orthogonal decomposition of the $W$-isotypic component of $U$ if and only if $T_{1}, T_{2}, \ldots, T_{m}$ form an orthonormal basis for $\operatorname{Hom}_{G}(W, U)$. Moreover, if this is the case, we then have:

$$
T_{j}^{*} T_{i}=\delta_{i, j} I_{W}
$$

for all $i, j=1,2, \ldots, m$.

Proof. Suppose that $T_{1}, T_{2}, \ldots, T_{m}$ form an orthonormal basis for $\operatorname{Hom}_{G}(W, U)$. Taking into account (2.2) we have $T_{j}^{*} T_{i} \in \operatorname{Hom}_{G}(W, W)$. By irreducibility we deduce from Schur's lemma that there exist $\lambda_{i, j} \in \mathbb{C}$ such that $T_{j}^{*} T_{i}=\lambda_{i, j} I_{W}$ for all $i, j=1,2, \ldots, m$. By taking traces, we get $\delta_{i, j} d_{\sigma}=\operatorname{tr}\left(\mathrm{T}_{\mathrm{j}}^{*} \mathrm{~T}_{\mathrm{i}}\right)=\lambda_{\mathrm{i}, \mathrm{j}} \mathrm{d}_{\sigma} \Rightarrow \lambda_{\mathrm{i}, \mathrm{j}}=\delta_{\mathrm{i}, \mathrm{j}}$ and (2.3) and (2.4) follow. The converse implication is trivial.

We denote by $L(G)$ the group algebra of $G$. This is the vector space of all functions $f: G \rightarrow \mathbb{C}$ equipped with the convolution product $*$ defined by setting $\left[f_{1} * f_{2}\right](g)=$ $\sum_{h \in G} f_{1}(h) f_{2}\left(h^{-1} g\right)=\sum_{h \in G} f_{1}(g h) f_{2}\left(h^{-1}\right)$ for all $f_{1}, f_{2} \in L(G)$ and $g \in G$. We shall endow $L(G)$ with the scalar product $\langle\cdot, \cdot\rangle_{L(G)}$ defined by setting

$$
\left\langle f_{1}, f_{2}\right\rangle_{L(G)}=\sum_{g \in G} f_{1}(g) \overline{f_{2}(g)}
$$

for all $f_{1}, f_{2} \in L(G)$.

Let $(\sigma, W)$ be a representation of $G$ and let $\left\{w_{1}, w_{2}, \ldots, w_{d_{\sigma}}\right\}$ be an orthonormal basis of $W$. The corresponding matrix coefficients $u_{j, i} \in L(G)$ are defined by setting

$$
u_{j, i}(g)=\left\langle\sigma(g) w_{i}, w_{j}\right\rangle
$$

for all $i, j=1,2, \ldots, d_{\sigma}$ and $g \in G$.

Proposition 2.2. Let $\sigma, \rho \in \widehat{G}$. Then

$$
\left\langle u_{i, j}^{\sigma}, u_{h, k}^{\rho}\right\rangle=\frac{|G|}{d_{\sigma}} \delta_{\sigma, \rho} \delta_{i, h} \delta_{j, k} \text { (orthogonality relations) }
$$

and

$$
u_{i, j}^{\sigma} * u_{h, k}^{\rho}=\frac{|G|}{d_{\sigma}} \delta_{\sigma, \rho} \delta_{j, h} u_{i, k}^{\sigma} \text { (convolution properties) }
$$

for all $i, j=1,2, \ldots, d_{\sigma}$ and $h, k=1,2, \ldots, d_{\rho}$.

Proof. See Lemma 3.6.3 and Lemma 3.9.14 of [2].

The sum $\chi_{\sigma}=\sum_{i=1}^{d_{\sigma}} u_{i, i} \in L(g)$ of the diagonal entries of matrix coefficients is called the character of $\sigma$.

Given a vector space $V$, we denote by $V^{\prime}=\operatorname{Hom}(V, \mathbb{C})$ its dual space. The conjugate of a linear operator $T \in \operatorname{Hom}(V, V)$ is the linear operator $T^{\prime} \in \operatorname{Hom}\left(V^{\prime}, V^{\prime}\right)$ defined by $\left[T^{\prime}\left(v^{\prime}\right)\right](v)=v^{\prime}(T(v))$ for all $v^{\prime} \in V^{\prime}$ and $v \in V$. The conjugate of a $G$-representation $(\sigma, W)$ is the $G$-representation $\left(\sigma^{\prime}, W^{\prime}\right)$ defined by $\sigma^{\prime}(g)=\left(\sigma\left(g^{-1}\right)\right)^{\prime}$ for all $g \in G$. Note that $d_{\sigma}=d_{\sigma^{\prime}}$ and that $\sigma$ is irreducible if and only if $\sigma^{\prime}$ is. Note that one may have $\sigma \neq \sigma^{\prime}$ but it is always the case that $\left(\sigma^{\prime}\right)^{\prime} \sim \sigma$. 
Let $G_{1}$ and $G_{2}$ be two finite groups and suppose that $\left(\sigma_{i}, W_{i}\right)$ is a $G_{i}$-representation for $i=1,2$. The outer tensor product of $\sigma_{1}$ and $\sigma_{2}$ is the $\left(G_{1} \times G_{2}\right)$-representation $\left(\sigma_{1} \otimes \sigma_{2}, W_{1} \otimes W_{2}\right)$ defined on simple tensors by setting $\left[\left(\sigma_{1} \otimes \sigma_{2}\right)\left(g_{1}, g_{2}\right)\right]\left(w_{1} \otimes w_{2}\right)=$ $\left(\sigma_{1}\left(g_{1}\right) w_{1}\right) \otimes\left(\sigma_{2}\left(g_{2}\right) w_{2}\right)$ for all $g_{i} \in G_{i}$ and $w_{i} \in W_{i}, i=1,2$ and then extending by linearity. When $G_{1}=G_{2}=G$, we denote by $\widetilde{G}=\{(g, g): g \in G\}$ the diagonal subgroup of $G \times G$ (this is clearly isomorphic to and we shall thus identify it with $G$ ) we set $\sigma_{1} \otimes \sigma_{2}=\operatorname{Res}_{G}^{G \times G}\left(\sigma_{1} \otimes \sigma_{2}\right)$ and call the $G$-representation $\left(\sigma_{1} \otimes \sigma_{2}, W_{1} \otimes W_{2}\right)$ the inner tensor product of $\sigma_{1}$ and $\sigma_{2}$.

In general, the inner tensor product of two irreducble $G$-representations is not irreducible: the following easy lemma, however, gives a sufficient condition guaranteeing its irreducibility (we include the proof for the readers' convenience).

Lemma 2.3. Let $G$ be a finite group and let $\sigma_{1}, \sigma_{2} \in \widehat{G}$. Suppose that $\operatorname{dim} \sigma_{1}=1$. Then $\sigma_{1} \otimes \sigma_{2}$ is irreducible.

Proof. We have

$$
\begin{aligned}
\left\langle\chi_{\sigma_{1} \otimes \sigma_{2}}, \chi_{\sigma_{1} \otimes \sigma_{2}}\right\rangle & =\left\langle\chi_{\sigma_{1}} \chi_{\sigma_{2}}, \chi_{\sigma_{1}} \chi_{\sigma_{2}}\right\rangle \\
& =\frac{1}{|G|} \sum_{g \in G} \chi_{\sigma_{1}}(g) \chi_{\sigma_{2}}(g) \overline{\chi_{\sigma_{1}}(g) \chi_{\sigma_{2}}(g)} \\
\text { (since } \left.\overline{\chi_{\sigma_{1}}(g)}=\chi_{\sigma_{1}}\left(g^{-1}\right)\right) & =\frac{1}{|G|} \sum_{g \in G} \chi_{\sigma_{1}}(g) \chi_{\sigma_{1}}\left(g^{-1}\right) \chi_{\sigma_{2}}(g) \overline{\chi_{\sigma_{2}}(g)} \\
\text { (since } \chi_{\sigma_{1}} \sim \sigma_{1} \text { is multiplicative) } & =\frac{1}{|G|} \sum_{g \in G} \chi_{\sigma_{1}}\left(g g^{-1}\right) \chi_{\sigma_{2}}(g) \overline{\chi_{\sigma_{2}}(g)} \\
& =\frac{1}{|G|} \sum_{g \in G} \chi_{\sigma_{2}}(g) \overline{\chi_{\sigma_{2}}(g)} \\
& =1 .
\end{aligned}
$$

Alternatively, up to identifying $V_{\sigma_{1} \otimes \sigma_{2}}$ with $V_{\sigma_{2}}$, which we simply denote by $V$, suppose that $W$

subseteq $V$ is a $\sigma_{1} \otimes \sigma_{2}$ invariant subspace and let $g \in G$. We then have $\sigma_{1}(g) \sigma_{2}(g) W=$ $\left(\sigma_{1} \otimes \sigma_{2}\right)(g) W \subseteq W$ and, by multiplying on the left by the scalar $\overline{\sigma_{1}(g)}$, we obtain $\sigma_{2}(g) W \subseteq \overline{\sigma_{1}(g)} W=W$. Thus $W$

subsete $V$ is $\sigma_{2}$ invariant and since $\sigma_{2}$ is irreducible, $W$ is trivial.

Suppose that $G$ acts transitively on a finite set $X$ and denote by $L(X)$ the vector space of all functions $f: X \rightarrow \mathbb{C}$. The associated permutation representation $(\lambda, L(X))$ is defined by $[\lambda(g) f](x)=f\left(g^{-1} x\right)$ for all $f \in L(X), x \in X$ and $g \in G$.

Fix a point $x_{0} \in X$ and denote by $K=\left\{g \in G: g x_{0}=x_{0}\right\} \leq G$ its stabilizer. Then we may identify $X$ with the set $G / K$ of left cosets of $K$ in $G$ as homogeneous $G$-spaces and $L(X)$ with the $L(G)$-subalgebra $L(G)^{K}=\{f \in L(G): f(g k)=f(g) \forall g \in G, \forall k \in K\}$ of $K$-right-invariant functions. Moreover we denote by ${ }^{K} L(G)^{K}=\left\{f \in L(G): f\left(k_{1} g k_{2}\right)=\right.$ $\left.f(g) \forall g \in G, \forall k_{1}, k_{2} \in K\right\}$ the subalgebra of bi-K-invariant functions.

Given a representation $(\sigma, W)$ we denote by $W^{K}=\{w \in W: \sigma(k) w=w, \forall k \in K\} \leq$ $W$ the subspace of $K$-invariant vectors of $W$. 
For the following result we refer to [1] and/or to the monographs [2, Chapter 4] and [5].

Theorem 2.4. The following conditions are equivalent:

(a) The algebra ${ }^{K} L(G)^{K}$ is commutative;

(b) the permutation representation $(\lambda, L(X))$ is multiplicity-free;

(c) the algebra $\operatorname{Hom}_{G}(L(X), L(X))$ is commutative;

(d) for every $(\sigma, W) \in \widehat{G}$ one has $\operatorname{dim}\left(W^{K}\right) \leq 1$;

(e) for every $(\sigma, W) \in \widehat{G}$ one has $\operatorname{dimHom}_{G}(W, L(X)) \leq 1$.

Definition 2.5. If one of the equivalent conditions in Theorem 2.4 is satisfied, on says that $(G, K)$ is a Gelfand pair.

The equivalence $(\mathrm{d}) \Leftrightarrow(\mathrm{e})$ in Theorem 2.4 is a particular case of the fact that the multiplicity of $(\sigma, W)$ in $(\lambda, L(X))$ is equal to $\operatorname{dim} W^{K}$. More precisely (see [3, Section 1.2.1] or [6]), with every $w \in W^{K}$ we may associate the linear map $T_{w}: W \rightarrow L(X)$ defined by setting

$$
\left(T_{w} v\right)(x)=\sqrt{\frac{d_{\sigma}}{|X|}}\langle v, \sigma(g) w\rangle_{V}
$$

for all $v \in W, x \in X$ (here $g$ is any element in $G$ such that $g x_{0}=x$ ). Then one easily checks that $T_{w} \in \operatorname{Hom}_{G}(W, L(X))$ and that

$$
\left\langle T_{w_{1}} v_{1}, T_{w_{2}} w_{2}\right\rangle_{L(X)}=\left\langle v_{1}, v_{2}\right\rangle_{W}\left\langle w_{2}, w_{1}\right\rangle_{W}
$$

for all $w_{1}, w_{2}, v_{1}, v_{2} \in W$. This easily implies that the map

$$
w \longrightarrow T_{w}
$$

yields an isometry between $W^{K}$ and $\operatorname{Hom}_{G}(W, L(X))$.

An irreducible representation $(\sigma, W) \in \widehat{G}$ contained in $(\lambda, L(X))$ is called spherical and its spherical character is the function $\chi_{\sigma}^{K} \in L(G)$ defined by

$$
\chi_{\sigma}^{K}(g)=\frac{1}{|K|} \sum_{k \in K} \overline{\chi_{\sigma}(k g)}
$$

for all $g \in G$, where $\chi_{\sigma}$ denotes the (usual) character of $\sigma$ (see [3, Section 1.2.2] or [6]). In particular,

$$
\chi_{\sigma}^{K}\left(1_{G}\right)=\text { multiplicity of } \sigma \text { in } \lambda .
$$

\section{Harmonic anAlysis FOR the pair $(G \times G \times H, \tilde{H})$}

Let $G$ be a finite group and $H \leq G$ be a subgroup.

We define a transitive action of the group $G \times G \times H$ on the set $X=G \times G$ by setting

$$
\left(g_{1}, g_{2}, h\right)\left(g_{3}, g_{4}\right)=\left(g_{1} g_{3} g_{2}^{-1}, g_{2} g_{4} h^{-1}\right)
$$

for all $\left(g_{1}, g_{2}, h\right) \in G \times G \times H$ and $\left(g_{3}, g_{4}\right) \in G \times G$. The stabilizer of $\left(1_{G}, 1_{G}\right) \in G \times G$ is the subgroup $\widetilde{H}=\{(h, h, h): h \in H\}$. Denote by $\eta$ the associated permutation representation of $G \times G \times H$, so that

$$
\left[\eta\left(g_{1}, g_{2}, h\right) f\right]\left(g_{3}, g_{4}\right)=f\left(g_{1}^{-1} g_{3} g_{2}, g_{2}^{-1} g_{4} h\right)
$$

for all $f \in L(G \times G),\left(g_{1}, g_{2}, h\right) \in G \times G \times H$ and $\left(g_{3}, g_{4}\right) \in G \times G$. 
3.1. A Frobenius reciprocity type theorem and proof of the main result. We shall deduce Theorem 1.1 from the following result which can be regarded as a sort of Frobenius reciprocity for permutation representations.

Theorem 3.1. Let $\left(\rho_{1}, V_{1}\right)$ and $\left(\rho_{2}, V_{2}\right)$ be two irreducible $G$-representations and let $(\theta, W)$ be an $H$-representation. Then the map

$$
\begin{array}{ccc}
\operatorname{Hom}_{G \times G \times H}\left(\rho_{1} \otimes \rho_{2} \otimes \theta, \eta\right) & \longrightarrow \operatorname{Hom}_{H}\left(\operatorname{Res}_{H}^{G}\left(\rho_{1} \otimes \rho_{2}\right), \theta^{\prime}\right) \\
T & \longmapsto & \widetilde{T}
\end{array}
$$

where

$$
\left[\widetilde{T}\left(v_{1} \otimes v_{2}\right)\right](w)=\frac{|G|}{\sqrt{d_{\theta}}}\left[T\left(v_{1} \otimes v_{2} \otimes w\right)\right]\left(1_{G}, 1_{G}\right)
$$

for all $v_{1} \in V_{1}, v_{2} \in V_{2}$ and $w \in W$, is an isometric isomorphism of vector spaces. Its inverse is given by the map

$$
\begin{array}{ccc}
\operatorname{Hom}_{H}\left(\operatorname{Res}_{H}^{G}\left(\rho_{1} \otimes \rho_{2}\right), \theta^{\prime}\right) & \longrightarrow & \operatorname{Hom}_{G \times G \times H}\left(\rho_{1} \otimes \rho_{2} \otimes \theta, \eta\right) \\
S & \longmapsto & \widehat{S}
\end{array}
$$

where

$$
\left[\widehat{S}\left(v_{1} \otimes v_{2} \otimes w\right)\right]\left(g_{1}, g_{2}\right)=\frac{\sqrt{d_{\theta}}}{|G|}\left\{S\left[\rho_{1}\left(g_{2}^{-1} g_{1}^{-1}\right) v_{1} \otimes \rho_{2}\left(g_{2}^{-1}\right) v_{2}\right]\right\}(w)
$$

for all $v_{1} \in V_{1}, v_{2} \in V_{2}, w \in W$ and $g_{1}, g_{2} \in G$.

Proof. First of all, note that a linear operator $T: V_{1} \otimes V_{2} \otimes W \rightarrow L(G \times G)$ intertwines $\rho_{1} \otimes \rho_{2} \otimes \theta$ with $\eta$ if and only if

$$
\left\{T\left[\rho_{1}\left(g_{1}\right) v_{1} \otimes \rho_{2}\left(g_{2}\right) v_{2} \otimes \theta(h) w\right]\right\}\left(g_{3}, g_{4}\right)=\left[T\left(v_{1} \otimes v_{2} \otimes w\right)\right]\left(g_{1}^{-1} g_{3} g_{2}, g_{2}^{-1} g_{4} h\right),
$$

while a linear operator $S: V_{1} \otimes V_{2} \rightarrow W^{\prime}$ intertwines $\operatorname{Res}_{H}^{G}\left(\rho_{1} \otimes \rho_{2}\right)$ with $\theta^{\prime}$ if and only if

$$
\left\{S\left[\rho_{1}(h) v_{1} \otimes \rho_{2}(h) v_{2}\right]\right\}(w)=\left[\theta^{\prime}(h) S\left(v_{1} \otimes v_{2}\right)\right](w),
$$

for all $v_{1} \in V_{1}, v_{2} \in V_{2}, w \in W, g_{1}, g_{2}, g_{3}, g_{4} \in G$ and $h \in H$. It follows that:

$$
\begin{aligned}
\left\{\widetilde{T}\left[\rho_{1}(h) v_{1} \otimes \rho_{2}(h) v_{2}\right]\right\}(w) & =\left\{T\left[\rho_{1}(h) v_{1} \otimes \rho_{2}(h) v_{2} \otimes w\right]\right\}\left(1_{G}, 1_{G}\right) \\
(\text { by }(\underline{3.2})) & =\frac{|G|}{\sqrt{d_{\theta}}}\left\{T\left[v_{1} \otimes v_{2} \otimes w\right]\right\}\left(1_{G}, h^{-1}\right) \\
(\text { again by (3.2) })) & =\frac{|G|}{\sqrt{d_{\theta}}}\left\{T\left[v_{1} \otimes v_{2} \otimes \theta\left(h^{-1}\right) w\right]\right\}\left(1_{G}, 1_{G}\right) \\
& =\left[\widetilde{T}\left(v_{1} \otimes v_{2}\right)\right]\left(\theta\left(h^{-1}\right) w\right) \\
& =\left[\theta^{\prime}(h) \widetilde{T}\left(v_{1} \otimes v_{2}\right)\right](w) .
\end{aligned}
$$


That is, $\widetilde{T}$ intertwines $\operatorname{Res}_{H}^{G}\left(\rho_{1} \otimes \rho_{2}\right)$ with $\theta^{\prime}$. Similarly,

$$
\begin{aligned}
\left\{\widehat{S}\left[\rho_{1}\left(g_{1}\right) v_{1} \otimes \rho_{2}\left(g_{2}\right) v_{2} \otimes \theta(h) w\right]\right\}\left(g_{3}, g_{4}\right) & =\frac{\sqrt{d_{\theta}}}{|G|}\left\{S\left[\rho_{1}\left(g_{4}^{-1} g_{3}^{-1} g_{1}\right) v_{1} \otimes \rho_{2}\left(g_{4}^{-1} g_{2}\right) v_{2}\right]\right\}(\theta(h) w) \\
& =\frac{\sqrt{d_{\theta}}}{|G|}\left\{\theta^{\prime}\left(h^{-1}\right) S\left[\rho_{1}\left(g_{4}^{-1} g_{3}^{-1} g_{1}\right) v_{1} \otimes \rho_{2}\left(g_{4}^{-1} g_{2}\right) v_{2}\right]\right\}(w) \\
(\text { by (莓.3) })) & =\frac{\sqrt{d_{\theta}}}{|G|}\left\{S\left[\rho_{1}\left(h^{-1} g_{4}^{-1} g_{3}^{-1} g_{1}\right) v_{1} \otimes \rho_{2}\left(h^{-1} g_{4}^{-1} g_{2}\right) v_{2}\right]\right\}(w) \\
& =\left[\widehat{S}\left(v_{1} \otimes v_{2} \otimes w\right)\right]\left(g_{1}^{-1} g_{3} g_{2}, g_{2}^{-1} g_{4} h\right)
\end{aligned}
$$

and therefore $\widehat{S}$ intertwines $\rho_{1} \otimes \rho_{2} \otimes \theta$ with $\eta$. Moreover, we have

$$
\begin{aligned}
{\left[\widehat{\widetilde{T}}\left(v_{1} \otimes v_{2} \otimes w\right)\right]\left(g_{1}, g_{2}\right) } & =\frac{\sqrt{d_{\theta}}}{|G|}\left\{\widetilde{T}\left[\rho_{1}\left(g_{2}^{-1} g_{1}^{-1}\right) v_{1} \otimes \rho_{2}\left(g_{2}^{-1}\right) v_{2}\right]\right\}(w) \\
& =\left\{T\left[\rho_{1}\left(g_{2}^{-1} g_{1}^{-1}\right) v_{1} \otimes \rho_{2}\left(g_{2}^{-1}\right) v_{2} \otimes w\right]\right\}\left(1_{G}, 1_{G}\right) \\
(\text { by (3.2) })) & =\left[T\left(v_{1} \otimes v_{2} \otimes w\right)\right]\left(g_{1}, g_{2}\right)
\end{aligned}
$$

and

$$
\begin{aligned}
{\left[\widetilde{\widehat{S}}\left(v_{1} \otimes v_{2}\right)\right](w) } & =\frac{|G|}{\sqrt{d_{\theta}}}\left[\widehat{S}\left[\left(v_{1} \otimes v_{2} \otimes w\right)\right]\left(1_{G}, 1_{G}\right)\right. \\
& =\left[S\left(v_{1} \otimes v_{2}\right)\right](w),
\end{aligned}
$$

that is, $\widehat{\widetilde{T}}=T$ and $\widetilde{\widehat{S}}=S$. It follows that the map $T \mapsto \widetilde{T}$ is a bijection and its inverse is $S \mapsto \widehat{S}$. Finally for $i=1,2$ let $\left\{v_{i, j}: j=1,2, \ldots, d_{\rho_{i}}\right\}$ be an orthonormal basis in $V_{i}$ and denote by $u_{h, j}^{\rho_{i}}$ the associated matrix coefficients; let also $\left\{w_{j}: j=1,2, \ldots, d_{\theta}\right\}$ be an orthonormal basis in $W$. If $T_{1}, T_{2} \in \operatorname{Hom}_{G \times G \times H}\left(\rho_{1} \otimes \rho_{2} \otimes \theta, \eta\right)$ then we have, on the one hand,

$$
\begin{aligned}
& \left\langle\widetilde{T}_{1}, \widetilde{T}_{2}\right\rangle=\frac{1}{d_{\rho_{1}} d_{\rho_{2}}} \operatorname{tr}\left(\widetilde{\mathrm{T}}_{2}^{*} \widetilde{\mathrm{T}}_{1}\right) \\
& =\frac{1}{d_{\rho_{1}} d_{\rho_{2}}} \sum_{i=1}^{d_{\rho_{1}}} \sum_{j=1}^{d_{\rho_{2}}}\left\langle\widetilde{T}_{1}\left(v_{1, i} \otimes v_{2, j}\right), \widetilde{T}_{2}\left(v_{1, i} \otimes v_{2, j}\right)\right\rangle_{W^{\prime}} \\
& =\frac{1}{d_{\rho_{1}} d_{\rho_{2}}} \sum_{i=1}^{d_{\rho_{1}}} \sum_{j=1}^{d_{\rho_{2}}} \sum_{\ell=1}^{d_{\theta}}\left[\widetilde{T}_{1}\left(v_{1, i} \otimes v_{2, j}\right)\right]\left(w_{\ell}\right) \cdot \overline{\left[\widetilde{T}_{2}\left(v_{1, i} \otimes v_{2, j}\right)\right]\left(w_{\ell}\right)} \\
& =\frac{|G|^{2}}{d_{\rho_{1}} d_{\rho_{2}} d_{\theta}} \sum_{h=1}^{d_{\rho_{1}}} \sum_{m=1}^{d_{\rho_{2}}} \sum_{\ell=1}^{d_{\theta}}\left[T_{1}\left(v_{1, h} \otimes v_{2, m} \otimes w_{\ell}\right)\right]\left(1_{G}, 1_{G}\right) \text {. } \\
& \cdot \overline{\left[T_{2}\left(v_{1, h} \otimes v_{2, m} \otimes w_{\ell}\right)\right]\left(1_{G}, 1_{G}\right)}
\end{aligned}
$$


and, on the other hand,

$$
\begin{aligned}
& \left\langle T_{1}, T_{2}\right\rangle=\frac{1}{d_{\rho_{1}} d_{\rho_{2}} d_{\theta}} \sum_{i=1}^{d_{\rho_{1}}} \sum_{j=1}^{d_{\rho_{2}}} \sum_{\ell=1}^{d_{\theta}} \sum_{g_{1}, g_{2} \in G}\left[T_{1}\left(v_{1, i} \otimes v_{2, j} \otimes w_{\ell}\right)\right]\left(g_{1}, g_{2}\right) . \\
& \cdot \overline{\left[T_{2}\left(v_{1, i} \otimes v_{2, j} \otimes w_{\ell}\right)\right]\left(g_{1}, g_{2}\right)}
\end{aligned}
$$

$($ by $(\underline{3.2}))=\frac{1}{d_{\rho_{1}} d_{\rho_{2}} d_{\theta}} \sum_{i=1}^{d_{\rho_{1}}} \sum_{j=1}^{d_{\rho_{2}}} \sum_{\ell=1}^{d_{\theta}} \sum_{g_{1}, g_{2} \in G}\left[T_{1}\left(\rho_{1}\left(g_{2}^{-1} g_{1}^{-1}\right) v_{1, i} \otimes \rho_{2}\left(g_{2}^{-1}\right) v_{2, j} \otimes w_{\ell}\right)\right]\left(1_{G}, 1_{G}\right)$.

$$
\begin{gathered}
\cdot \overline{\left[T_{2}\left(\rho_{1}\left(g_{2}^{-1} g_{1}^{-1}\right) v_{1, i} \otimes \rho_{2}\left(g_{2}^{-1}\right) v_{2, j} \otimes w_{\ell}\right)\right]\left(1_{G}, 1_{G}\right)} \\
=\frac{1}{d_{\rho_{1}} d_{\rho_{2}} d_{\theta}} \sum_{i, h, s=1}^{d_{\rho_{1}}} \sum_{j, m, t=1}^{d_{\rho_{2}}} \sum_{\ell=1}^{d_{\theta}} \sum_{g_{2} \in G} u_{m, j}^{\rho_{2}}\left(g_{2}^{-1}\right) \overline{u_{t, j}^{\rho_{2}}\left(g_{2}^{-1}\right)} \sum_{g_{1} \in G} u_{h, i}^{\rho_{1}}\left(g_{2}^{-1} g_{1}^{-1}\right) \overline{u_{s, i}^{\rho_{1}}\left(g_{2}^{-1} g_{1}^{-1}\right)} \\
\\
\cdot\left[T_{1}\left(v_{1, h} \otimes v_{2, m} \otimes w_{\ell}\right)\right]\left(1_{G}, 1_{G}\right) \cdot \overline{\left[T_{2}\left(v_{1, s} \otimes v_{2, t} \otimes w_{\ell}\right)\right]\left(1_{G}, 1_{G}\right)}
\end{gathered}
$$

$($ by (2.7) $)=\frac{|G|^{2}}{d_{\rho_{1}} d_{\rho_{2}} d_{\theta}} \sum_{h=1}^{d_{\rho_{1}}} \sum_{m=1}^{d_{\rho_{2}}} \sum_{\ell=1}^{d_{\theta}}\left[T_{1}\left(v_{1, h} \otimes v_{2, m} \otimes w_{\ell}\right)\right]\left(1_{G}, 1_{G}\right)$.

$$
\cdot \overline{\left[T_{2}\left(v_{1, h} \otimes v_{2, m} \otimes w_{\ell}\right)\right]\left(1_{G}, 1_{G}\right)} .
$$

We deduce that $\left\langle\widetilde{T}_{1}, \widetilde{T}_{2}\right\rangle_{\operatorname{Hom}\left(V_{1} \otimes V_{2}, W^{\prime}\right)}=\left\langle T_{1}, T_{2}\right\rangle_{\operatorname{Hom}\left(V_{1} \otimes V_{2} \otimes W, L(G \times G)\right)}$ showing that the bijective map $T \mapsto \widetilde{T}$ is indeed an isometry.

Proof of Theorem 1.1. We first observe that if an $H$-representation $\theta$ is irreducible, then the multiplicity of the irreducible $(G \times G \times H)$-representation $\rho_{1} \otimes \rho_{2} \otimes \theta$ in the permutation representation $\eta$ (associated with the pair $(G \times G \times H, \widetilde{H}))$ equals the multiplicity of the irreducible $H$-representation $\theta^{\prime}$ in $\operatorname{Res}_{H}^{G}\left(\rho_{1} \otimes \rho_{2}\right)$. Moreover, all irreducible $(G \times G \times H)-$ (resp. $H$-)representations are, up to equivalence, equal to $\rho_{1} \otimes \rho_{2} \otimes \theta$ (resp. $\theta^{\prime}$ ) with $\rho_{1}, \rho_{2} \in$ $\widehat{G}$ and $\theta \in \widehat{H}$. Theorefore $\eta$ is multiplicity-free (and therefore, all equivalent conditions in Theorem 2.4 are satisfied for the pair $(G \times G \times H, \widetilde{H}))$ if and only if $\operatorname{Res}_{H}^{G}\left(\rho_{1} \otimes \rho_{2}\right)$ is multiplicity-free.

3.2. Invariant vectors and spherical characters. In this section we describe the isometry (2.9) and the spherical characters for the pair $(G \times G \times H, \widetilde{H})$.

Using the description of tensor products in [2, Section 9.1] (see also [7]), we identify $V_{1} \otimes V_{2} \otimes W$ with the vector space of all anti-trilinear maps $B: V_{1} \times V_{2} \times W \rightarrow \mathbb{C}$. Moreover, an element $B \in V_{1} \otimes V_{2} \otimes W$ is $\widetilde{H}$-invariant (that is, $B \in\left(V_{1} \otimes V_{2} \otimes W\right)^{\widetilde{H}}$ ) if and only if

$$
B\left(\rho_{1}(h) v_{1}, \rho_{2}(h) v_{2}, \theta(h) w\right)=B\left(v_{1}, v_{2}, w\right)
$$

for all $v_{1} \in V_{1}, v_{2} \in V_{2}, w \in W$ and $h \in H$. 
Proposition 3.2. Let $B \in\left(V_{1} \otimes V_{2} \otimes W\right)^{\widetilde{H}}$. Then the associated intertwing operator $T_{B} \in \operatorname{Hom}_{G \times G \times H}\left(\rho_{1} \otimes \rho_{2} \otimes \theta, \eta\right)$ given by (2.9) has the form:

$$
\left[T_{B}\left(v_{1} \otimes v_{2} \otimes w\right)\right]\left(g_{1}, g_{2}\right)=\frac{\sqrt{d_{\rho_{1}} d_{\rho_{2}} d_{\theta}}}{|G|} \overline{B\left(\rho_{1}\left(g_{2}^{-1} g_{1}^{-1}\right) v_{1}, \rho_{2}\left(g_{2}^{-1}\right) v_{2}, w\right)} .
$$

for all $v_{1} \in V_{1}, v_{2} \in V_{2}$ and $w \in W$.

Proof. First note that

$$
\left\langle v_{1} \otimes v_{2} \otimes w, B\right\rangle=\overline{\left\langle B, v_{1} \otimes v_{2} \otimes w\right\rangle}=\overline{B\left(v_{1}, v_{2}, w\right\rangle}
$$

and that the last expression is trilinear. Moreover, recalling (3.1), we have $\left(g_{3}, g_{4}, h\right)\left(1_{G}, 1_{G}\right)=$ $\left(g_{1}, g_{2}\right)$ if we take $g_{3}=g_{1} g_{2}, g_{4}=g_{2}$ and $h=1_{G}$. By virtue of these simple considerations we may write (2.9) explicitely as follows:

$$
\begin{aligned}
{\left[T_{B}\left(v_{1} \otimes v_{2} \otimes w\right)\right]\left(g_{1}, g_{2}\right) } & =\frac{\sqrt{d_{\rho_{1}} d_{\rho_{2}} d_{\theta}}}{|G|}\left\langle v_{1} \otimes v_{2} \otimes w,\left[\rho_{1}\left(g_{1} g_{2}\right) \otimes \rho_{2}\left(g_{2}\right) \otimes \theta\left(1_{G}\right)\right] B\right\rangle \\
& =\frac{\sqrt{d_{\rho_{1}} d_{\rho_{2}} d_{\theta}}}{|G|}\left\langle\rho_{1}\left(g_{2}^{-1} g_{1}^{-1}\right) v_{1} \otimes \rho_{2}\left(g_{2}^{-1}\right) v_{2} \otimes w, B\right\rangle \\
& =\frac{\sqrt{d_{\rho_{1}} d_{\rho_{2}} d_{\theta}}}{|G|} \frac{\left.1 \rho_{1}\left(g_{2}^{-1} g_{1}^{-1}\right) v_{1}, \rho_{2}\left(g_{2}^{-1}\right) v_{2}, w\right)}{B} .
\end{aligned}
$$

By combining the isometries in Theorem 3.1 and Proposition 3.2 we obtain the following.

Corollary 3.3. The map $B \mapsto \widetilde{T_{B}}$ yields an isometry

$$
\left(V_{1} \otimes V_{2} \otimes W\right)^{\widetilde{H}} \rightarrow \operatorname{Hom}_{H}\left(\operatorname{Res}_{H}^{G}\left(\rho_{1} \otimes \rho_{2}\right), \theta^{\prime}\right) .
$$

Moreover, for $B \in V_{1} \otimes V_{2} \otimes W$, the intertwining operator $\widetilde{T_{B}}$ has the form:

$$
\left[\widetilde{T_{B}}\left(v_{1} \otimes v_{2}\right)\right](w)=\sqrt{d_{\rho_{1}} d_{\rho_{2}}} \overline{B\left(v_{1}, v_{2}, w\right)}
$$

for all $v_{1} \in V_{1}, v_{2} \in V_{2}$ and $w \in W$.

Clearly, the last Corollary may be proved directly following the same arguments in the proof of Theorem 3.1 .

We end this section by providing an explicit expression of (2.10) for the pair $(G \times G \times$ $H, \widetilde{H})$. This quite easy: the spherical character associated to the for the pair $(G \times G \times H$ representation $\rho_{1} \otimes \rho_{2} \otimes \theta$ is given by:

$$
\begin{aligned}
\psi_{\rho_{1} \bowtie \rho_{2} \bowtie \theta}\left(g_{1}, g_{2}, h_{1}\right) & =\frac{1}{|H|} \sum_{h \in H} \overline{\chi_{\rho_{1} \bowtie \rho_{2} \otimes \theta}\left(h g_{1}, h g_{2}, h h_{1}\right)} \\
& =\frac{1}{|H|} \sum_{h \in H} \overline{\chi_{\rho_{1}}\left(h g_{1}\right)} \overline{\chi_{\rho_{2}}\left(h g_{2}\right)} \overline{\chi_{\theta}\left(h h_{1}\right)}
\end{aligned}
$$


for all $g_{1}, g_{2} \in G$ and $h_{1} \in H$. In particular, (2.11) and the isomorphisms above yield

$$
\begin{aligned}
\psi_{\rho_{1} \bowtie \rho_{2} \otimes \theta}\left(1_{G}, 1_{G}, 1_{G}\right) & =\operatorname{dim}\left(V_{1} \times V_{2} \times W\right)^{\widetilde{H}} \\
& =\operatorname{dimHom}_{G \times G \times H}\left(\rho_{1} \otimes \rho_{2} \otimes \theta, \eta\right) \\
& =\operatorname{dimHom}_{H}\left(\operatorname{Res}_{H}^{G}\left(\rho_{1} \otimes \rho_{2}\right), \theta^{\prime}\right) \\
& \equiv \text { multiplicity of } \theta^{\prime} \text { in } \operatorname{Res}_{H}^{G}\left(\rho_{1} \otimes \rho_{2}\right) .
\end{aligned}
$$

The fact that $\psi_{\rho_{1} \otimes \rho_{2} \otimes \theta}\left(1_{G}, 1_{G}, 1_{G}\right)$ equals the multiplicity of $\theta^{\prime}$ in $\operatorname{Res}_{H}^{G}\left(\rho_{1} \otimes \rho_{2}\right)$ could also be recovered from the explicit expression of $\psi_{\rho_{1} \unrhd \rho_{2} \bowtie \theta}\left(1_{G}, 1_{G}, 1_{G}\right)$ which is nothing but the scalar product between the character of $\theta^{\prime}$ and the character of $\operatorname{Res}_{H}^{G}\left(\rho_{1} \otimes \rho_{2}\right)$.

\section{Harmonic ANALYSis on ClifFord groups}

In this section we consider the Clifford groups $G=\mathbb{C L}(n)$ and we study the decomposition of the tensor product of two irreducible representations and its restriction to the subgroup $H=\mathbb{C L}(n-1)$.

4.1. Clifford groups and their irreducible representations. Let $n \in \mathbb{N}$ and set $X_{n}=\{1,2, \ldots, n\}$. Denote by $\mathbb{C L}(n)$ the Clifford group of degree $n$. Recall that $\mathbb{C L}(n)=$ $\left\{ \pm \gamma_{A}: A \subseteq X_{n}\right\}$ with multiplication given by

$$
\varepsilon_{1} \gamma_{A} \cdot \varepsilon_{2} \gamma_{B}=\varepsilon_{1} \varepsilon_{2}(-1)^{\xi(A, B)} \gamma_{A \triangle B}
$$

where $\triangle$ denotes the symmetric difference of two sets and $\xi(A, B)$ equals the number of elements $(a, b) \in A \times B$ such that $a>b$, for all $\varepsilon_{1}, \varepsilon_{2} \in\{1,-1\}$ and $A, B \subseteq X_{n}$. Notice that the identity element is given by $1=\gamma_{\varnothing}$ and that $\left(\varepsilon \gamma_{A}\right)^{-1}=\varepsilon(-1)^{\frac{|A|(|A|-1)}{2}} \gamma_{A}$ for all $\varepsilon= \pm 1$ and $A \subseteq X_{n}$.

It is well known (cf. [7, Section IV.3]) that $\mathbb{C L}(n)$ admits exactly $2^{n}$ one-dimensional representations, namely $\chi_{A}, A \subseteq X_{n}$ given by

$$
\chi_{A}\left( \pm \gamma_{B}\right)=(-1)^{|A \cap B|}
$$

for all $B \subseteq X_{n}$, and, if $n$ is even, there is only one irreducible representation $\rho_{n}$, of dimension $2^{n / 2}$ whose character is given by

$$
\chi_{\rho_{n}}\left( \pm \gamma_{B}\right)= \pm \delta_{B, \varnothing} 2^{n / 2}
$$

for all $B \subseteq X_{n}$, and, if $n$ is odd, say $n=2 m+1$, there are exactly two irreducible representation $\rho_{n}^{ \pm}$, of dimension $2^{m}$ whose characters are given by

$$
\chi_{\rho_{n}^{ \pm}}\left(\gamma_{B}\right)= \begin{cases}2^{m} & \text { if } B=\varnothing \\ 0 & \text { if } B \neq \varnothing, X_{n} \\ \pm c 2^{m} & \text { if } B=X_{n}\end{cases}
$$

where

$$
c=\left\{\begin{array}{lll}
1 & \text { if } m \equiv 0 & \bmod 2 \\
-i & \text { if } m \equiv 1 & \bmod 2
\end{array}\right.
$$


and

$$
\chi_{\rho_{n}^{ \pm}}\left(-\gamma_{B}\right)=-\chi_{\rho_{n}^{ \pm}}\left(\gamma_{B}\right)
$$

for all $B \subseteq X_{n}$.

4.2. Kronecker products of irreducible representations of $\mathbb{C} \mathbb{L}(n)$. In this section we study the decomposition of the Kronecker products of irreducible representations of $\mathbb{C L}(n)$ and of their restriction to the subgroup $\mathbb{C L}(n-1)$, yielding the proof of Theorem 1.2 .

Proof of Theorem 1.2. (i). By Corollary 1.1 for $H=G$, this is equivalent to prove that the tensor product of any two irreducible representations of $\mathbb{C L}(n)$ decomposes multiplicitly free. To this end, we distinguish the two cases corresponding to the parity of $n$.

Suppose first that $n$ is even. By virtue of Lemma 2.3 we only need to analyse the decomposition of $\rho_{n} \otimes \rho_{n}$. Suppose $\theta \in \widehat{\mathbb{C L}(n)}$. Then we have

$$
\begin{aligned}
\left\langle\chi_{\rho_{n} \otimes \rho_{n}}, \chi_{\theta}\right\rangle & =\frac{1}{2^{n+1}} \sum_{g \in \mathbb{C L}(n)} \chi_{\rho_{n}}(g)^{2} \overline{\chi_{\theta}(g)} \\
& =\frac{1}{2^{n+1}}\left[\chi_{\rho_{n}}(1)^{2} \overline{\chi_{\theta}(1)}+\chi_{\rho_{n}}(-1)^{2} \overline{\chi_{\theta}(-1)}\right] \\
& =\frac{1}{2^{n+1}}\left[2^{n} \overline{\chi_{\theta}(1)}+2^{n} \overline{\chi_{\theta}(-1)}\right] \\
& = \begin{cases}1 & \text { if } \theta=\chi_{A}, A \subseteq X_{n}, \text { by (4.1) } \\
0 & \text { if } \theta=\rho_{n}, \quad \text { by (4.2). }\end{cases}
\end{aligned}
$$

It follows that

$$
\rho_{n} \otimes \rho_{n}=\bigoplus_{A \subseteq X_{n}} \chi_{A} .
$$

Suppose now that $n$ is odd, say $n=2 m+1$. As before, we need only to analyze $\rho_{n}^{+} \otimes \rho_{n}^{+}$, $\rho_{n}^{+} \otimes \rho_{n}^{-}$and $\rho_{n}^{-} \otimes \rho_{n}^{-}$and show that they decompose multiplicity free. Suppose $\theta \in \widehat{\mathbb{C L}(n)}$. Then we have

$$
\begin{aligned}
& \left\langle\chi_{\rho_{n} \otimes \rho_{n}}, \chi_{\theta}\right\rangle=\frac{1}{2^{n+1}} \sum_{g \in \mathbb{C L}(n)} \chi_{\rho_{n}}(g)^{2} \overline{\chi_{\theta}(g)} \\
& =\frac{1}{2^{n+1}}\left[\chi_{\rho_{n}}(1)^{2} \overline{\chi_{\theta}(1)}+\chi_{\rho_{n}}(-1)^{2} \overline{\chi_{\theta}(-1)}+\right. \\
& \left.+\chi_{\rho_{n}}\left(\gamma_{X_{n}}\right)^{2} \overline{\chi_{\theta}\left(\gamma_{X_{n}}\right)}+\chi_{\rho_{n}}\left(-\gamma_{X_{n}}\right)^{2} \overline{\chi_{\theta}\left(-\gamma_{X_{n}}\right)}\right] \\
& =\frac{1}{2^{n+1}}\left[2^{2 m}\left(\overline{\chi_{\theta}(1)}+\overline{\chi_{\theta}(-1)}\right)+c^{2} 2^{2 m}\left(\overline{\left(\chi_{\theta}\left(\gamma_{X_{n}}\right)\right.}+\overline{\chi_{\theta}\left(-\gamma_{X_{n}}\right)}\right)\right]
\end{aligned}
$$

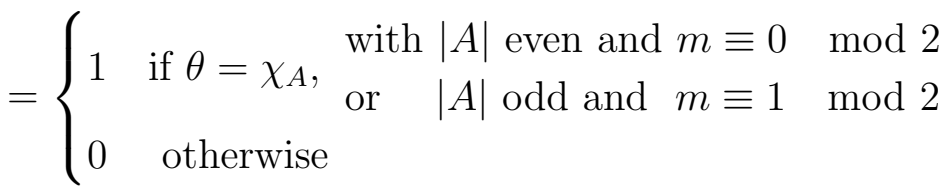


where the last equality follows from (4.2), (4.5) and (4.4). It follows that

$$
\rho_{n}^{+} \otimes \rho_{n}^{+}=\left\{\begin{array}{lll}
\bigoplus_{|A| \text { even } \chi_{A}} & \text { if } m \equiv 0 & \bmod 2 \\
\bigoplus_{|A| \text { odd }} \chi_{A} & \text { if } m \equiv 1 & \bmod 2 .
\end{array}\right.
$$

Similarly we have

$$
\rho_{n}^{+} \otimes \rho_{n}^{-}=\left\{\begin{array}{lll}
\bigoplus_{|A| \text { odd }} \chi_{A} & \text { if } m \equiv 0 & \bmod 2 \\
\bigoplus_{|A| \text { even } \chi_{A}} & \text { if } m \equiv 1 & \bmod 2
\end{array}\right.
$$

while

$$
\rho_{n}^{-} \otimes \rho_{n}^{-} \sim \rho_{n}^{+} \otimes \rho_{n}^{+}
$$

Proof of Theorem 1.2. (ii). By Corollary 1.1 this is equivalent to showing that the restriction to the subgroup $\mathbb{C L}(n-1)$ of the tensor product of any two irreducible representations of $\mathbb{C L}(n)$ decomposes multiplicitly free if and only if $n$ is odd.

We first observe that

$$
\chi_{A} \otimes \chi_{B}=\chi_{A \triangle B}
$$

and, if $n$ is even,

$$
\chi_{A} \otimes \rho_{n}=\rho_{n}
$$

while, if $n$ is odd,

$$
\chi_{A} \otimes \rho_{n}^{ \pm}= \begin{cases}\rho_{n}^{ \pm} & \text {if }|A| \text { is even } \\ \rho_{n}^{\mp} & \text { if }|A| \text { is odd }\end{cases}
$$

for all $A, B \subseteq X_{n}$.

Moreover,

$$
\operatorname{Res}_{\mathbb{C L}(n-1)}^{\mathbb{C L}(n)} \chi_{A}=\chi_{A \backslash\{n\}}
$$

for all $A \subseteq X_{n}$, and if $n$ is even,

$$
\operatorname{Res}_{\mathbb{C L}(n-1)}^{\mathbb{C L}(n)} \rho_{n}=\rho_{n-1}^{+} \oplus \rho_{n-1}^{-},
$$

while, if $n$ is odd,

$$
\operatorname{Res}_{\mathbb{C L}(n-1)}^{\mathbb{C L}(n)} \rho_{n}^{ \pm}=\rho_{n-1} .
$$

Collecting all these facts together, we deduce the following:

- $\operatorname{Res}_{\mathbb{C L}(n-1)}^{\mathbb{C L}(n)}\left(\chi_{A} \otimes \chi_{B}\right)=\chi_{(A \triangle B) \backslash\{n\}}$ (by (4.10) and (4.13) $)$;

- $\operatorname{Res}_{\mathbb{C L}(n-1)}^{\mathbb{L L}(n)}\left(\chi_{A} \otimes \rho_{n}\right)=\rho_{n-1}^{+} \oplus \rho_{n-1}^{-}$, if $n$ is even (by (4.11) and (4.14));

- $\operatorname{Res}_{\mathbb{C L}(n-1)}^{\mathbb{C L}(n)}\left(\chi_{A} \otimes \rho_{n}^{ \pm}\right)=\rho_{n-1}$, if $n$ is odd (by (4.12) and (4.15));

- $\operatorname{Res}_{\mathbb{C L}(n-1)}^{\mathbb{C L}(n)}\left(\rho_{n} \otimes \rho_{n}\right)=2 \bigoplus_{A \subseteq X_{n-1}} \chi_{A}$, if $n$ is even (by (4.6) and (4.13) );

- $\operatorname{Res}_{\mathbb{C L}(n-1)}^{\mathbb{C L}(n)}\left(\rho_{n-1}^{ \pm} \otimes \rho_{n-1}^{ \pm}\right)=\operatorname{Res}_{\mathbb{C L}(n-1)}^{\mathbb{C L}(n)}\left(\rho_{n-1}^{ \pm} \otimes \rho_{n-1}^{\mp}\right)=\bigoplus_{A \subseteq X_{n-1}} \chi_{A}$, if $n$ is odd (by (4.7), (4.8), (4.9) and (4.13)).

The proof is now complete. 
4.3. The orbits of $\mathbb{C L}(n)$ on $\mathbb{C L}(n) \times \mathbb{C L}(n)$. In this section we study the orbits of $\mathbb{C L}(n)$ acting by conjugation on the Cartesian product $\mathbb{C L}(n) \times \mathbb{C} \mathbb{L}(n)$. The interest for such analysis is motivated by the fact that spherical characters are constant on each orbit.

Let $A, C \subseteq X$. Then we have

$$
\begin{aligned}
\gamma_{C}^{-1} \gamma_{A} \gamma_{C} & =(-1)^{\frac{|C|(|C|-1)}{2}+\xi(A, C)+\xi(C, A \triangle C)} \gamma_{C \triangle(A \triangle C)} \\
& =(-1)^{\frac{|C|(|C|-1)}{2}+\xi(A, C)+\xi(C, A)+\xi(C, C)} \gamma_{A} \\
& ={ }_{*}(-1)^{|A||C|-|A \cap C|} \gamma_{A}
\end{aligned}
$$

where $={ }_{*}$ follows from the fact that $\xi(C, C)=\frac{|C|(|C|-1)}{2}$.

Theorem 4.1. Let $\emptyset \subseteq A, B \subseteq X_{n}$ and denote by $\mathcal{O}_{n}\left( \pm \gamma_{A}, \pm \gamma_{B}\right)$ the $\mathbb{C L}(n)$-orbit of $\left( \pm \gamma_{A}, \pm \gamma_{B}\right)$.

(a) $\mathcal{O}_{n}\left( \pm \gamma_{\emptyset}, \pm \gamma_{\emptyset}\right)=\left\{\left( \pm \gamma_{\emptyset}, \pm \gamma_{\emptyset}\right)\right\}$;

(b) if $A \neq B$ and $A, B \neq \emptyset, X_{n}$ then

$$
\mathcal{O}_{n}\left( \pm \gamma_{A}, \pm \gamma_{B}\right)=\left\{\left(\gamma_{A}, \gamma_{B}\right),\left(-\gamma_{A},-\gamma_{B}\right),\left(-\gamma_{A}, \gamma_{B}\right),\left(\gamma_{A},-\gamma_{B}\right)\right\}
$$

unless $n$ is odd and $A \amalg B=X_{n}$ (here and in the sequel $\coprod$ denotes a disjoint union) in which case

$$
\mathcal{O}_{n}\left( \pm \gamma_{A}, \pm \gamma_{B}\right)=\left\{\left( \pm \gamma_{A}, \pm \gamma_{B}\right),\left(\mp \gamma_{A}, \mp \gamma_{B}\right)\right\}
$$

(c) if $A \neq \emptyset$ then

$$
\mathcal{O}_{n}\left( \pm \gamma_{\emptyset}, \pm \gamma_{A}\right)=\left\{\left( \pm \gamma_{\emptyset}, \pm \gamma_{A}\right),\left( \pm \gamma_{\emptyset}, \mp \gamma_{A}\right)\right\}
$$

unless $n$ is odd and $A=X_{n}$, in which case $\mathcal{O}_{n}\left( \pm \gamma_{\emptyset}, \pm \gamma_{X_{n}}\right)=\left\{\left( \pm \gamma_{\emptyset}, \pm \gamma_{X_{n}}\right)\right\}$. The analogous result holds for $\mathcal{O}_{n}\left( \pm \gamma_{A}, \pm \gamma_{\emptyset}\right)$;

(d) if $A \neq \emptyset$ then

$$
\mathcal{O}_{n}\left( \pm \gamma_{A}, \pm \gamma_{A}\right)=\left\{\left( \pm \gamma_{A}, \pm \gamma_{A}\right),\left(\mp \gamma_{A}, \mp \gamma_{A}\right)\right\}
$$

unless $n$ is odd and $A=X_{n}$, in which case $\mathcal{O}_{n}\left( \pm \gamma_{X_{n}}, \pm \gamma_{X_{n}}\right)=\left\{\left( \pm \gamma_{X_{n}}, \pm \gamma_{X_{n}}\right)\right\}$.

Proof. We limit ourselves to prove (b) which is the most involved case: the remaining ones are simpler and can be treated in a similar manner. Suppose first that $|A|$ and $|B|$ are both even. We distinguish two cases. (i) None of $A$ and $B$ is contained in the other. Let then $a \in A \backslash B$ and $b \in B \backslash A$. Then taking $C=\{a, b\}$ (resp. $\{a\}$, resp. $\{b\}$ ) and using (4.16) gives $\gamma_{C}\left(\gamma_{A}, \gamma_{B}\right) \gamma_{C}^{-1}=\left(-\gamma_{A},-\gamma_{B}\right)$ (resp. $\left(-\gamma_{A}, \gamma_{B}\right)$, resp. $\left.\left(\gamma_{A},-\gamma_{B}\right)\right)$. (ii) Suppose $A$

subseteq $B$ (the specular case $B$ subseteq $A$ is treated in the same way) and let $a \in A$ and $b \in B \backslash A$. Then taking $C=\{a\}$ (resp. $\{a, b\}$, resp. $\{b\})$ and using (4.16) gives $\gamma_{C}\left(\gamma_{A}, \gamma_{B}\right) \gamma_{C}^{-1}=\left(-\gamma_{A},-\gamma_{B}\right)$ (resp. $\left(-\gamma_{A}, \gamma_{B}\right)$, resp. $\left.\left(\gamma_{A},-\gamma_{B}\right)\right)$. Thus (4.17) follows in both cases. If $|A|$ and $|B|$ are both odd, similar calculations yield again (4.17). We now consider the remaining case, namely when $|A|$ and $|B|$ have different parity. To fix ideas we suppose that $|A|$ is even and $|B|$ is odd. We distinguish three cases. (i) $A \cup B \neq X_{n}$ and $A \backslash B \neq \varnothing$. Let then $a \in A \backslash B$ and $c \in X_{n} \backslash(A \cup B)$. Then taking $C=\{a\}$ (resp. $\{a, c\}$, resp. $\{c\}$ ) and using (4.16) gives $\gamma_{C}\left(\gamma_{A}, \gamma_{B}\right) \gamma_{C}^{-1}=\left(-\gamma_{A},-\gamma_{B}\right)$ (resp. $\left(-\gamma_{A}, \gamma_{B}\right)$, resp. $\left.\left(\gamma_{A},-\gamma_{B}\right)\right)$. (ii) $A$ subseteq $B$. Let then $a \in A, b \in B \backslash A$ and $c \in X_{n} \backslash B$. Then taking $C=\{a, c\}$ (resp. $\{a\}$, 
resp. $\{b, c\})$ and using (4.16) gives $\gamma_{C}\left(\gamma_{A}, \gamma_{B}\right) \gamma_{C}^{-1}=\left(-\gamma_{A},-\gamma_{B}\right)\left(\right.$ resp. $\left(-\gamma_{A}, \gamma_{B}\right)$, resp. $\left.\left(\gamma_{A},-\gamma_{B}\right)\right)$. (iii) $A \cup B=X_{n}$. Suppose that $A \cap B \neq \varnothing$. Let then $a \in A \backslash B$ and $d \in A \cap B$ and set $C=\{a\}$ (resp. $\{d\}$, resp. $\{a, d\}$ ). We have $\gamma_{C}\left(\gamma_{A}, \gamma_{B}\right) \gamma_{C}^{-1}=\left(-\gamma_{A},-\gamma_{B}\right)$ (resp. $\left(-\gamma_{A}, \gamma_{B}\right)$, resp. $\left.\left(\gamma_{A},-\gamma_{B}\right)\right)$ and (4.17) follows also in this case. On the other hand, if $A \cap B=\varnothing$ (so that necessarily $n=|A|+|B|$ is odd) and $C \subseteq X_{n}$, then if $|C \cap A|$ is even (resp. odd) then $\gamma_{C}\left(\gamma_{A}, \gamma_{B}\right) \gamma_{C}^{-1}=\left(\gamma_{A}, \gamma_{B}\right)\left(\right.$ resp. $\left.\gamma_{C}\left(\gamma_{A}, \gamma_{B}\right) \gamma_{C}^{-1}=\left(-\gamma_{A},-\gamma_{B}\right)\right)$ and (4.18) follows.

4.4. The spherical characters for $(\mathbb{C L}(n) \times \mathbb{C L}(n) \times \mathbb{C L}(n), \widetilde{\mathbb{C L}(n)})$. In this section we study the spherical characters associated with the Gelfand pair $(\mathbb{C L}(n) \times \mathbb{C L}(n) \times$ $\mathbb{C L}(n), \widetilde{\mathbb{C L}(n)})$. We start with a simple preliminary combinatorial result. This could be immediately deduced from the orthogonality relations for the characters, but we prefer to give a direct proof.

Lemma 4.2. Let $U \subseteq X_{n}$. Then

$$
\frac{1}{2^{n}} \sum_{D \subseteq X_{n}}(-1)^{|U \cap D|}= \begin{cases}1 & \text { if } U=\varnothing \\ 0 & \text { otherwise. }\end{cases}
$$

Proof. If $U=\varnothing$, we have $|U \cap D|=\varnothing$ for every $D \subseteq X_{n}$ so that each summand in the Left Hand Side of (4.19) equals 1 and therefore the whole Left Hand Side of (4.19) equals 1. Suppose now that $U \neq \varnothing$ and fix $u \in U$. Consider the sets $\mathcal{P}_{0}=\left\{A \subseteq X_{n}\right.$ : $|A \cap U|$ is even $\}$ and $\mathcal{P}_{1}=\left\{A \subseteq X_{n}:|A \cap U|\right.$ is odd $\}$. Since the map $\Phi: \mathcal{P}_{0} \rightarrow \mathcal{P}_{1}$ defined by

$$
\Phi(A)= \begin{cases}A \backslash\{u\} & \text { if } u \notin A \\ A \cup\{u\} & \text { otherwise }\end{cases}
$$

is bijective, we have $\left|\mathcal{P}_{0}\right|=\left|\mathcal{P}_{1}\right|$. As a consequence,

$$
\frac{1}{2^{n}} \sum_{D \subseteq X_{n}}(-1)^{|U \cap D|}=\frac{1}{2^{n}}\left(\sum_{A \in \mathcal{P}_{0}}(-1)^{|U \cap D|}+\sum_{A \in \mathcal{P}_{1}}(-1)^{|U \cap D|}\right)=\frac{1}{2^{n}}\left(\left|\mathcal{P}_{0}\right|-\left|\mathcal{P}_{1}\right|\right)=0
$$

The spherical character on $\mathbb{C L}(n) \times \mathbb{C L}(n) \times \mathbb{C L}(n)$ associated with the representation $\chi_{A} \otimes \chi_{B} \otimes \chi_{C}$, for $A, B, C \subseteq X_{n}$, is given by

$$
\psi_{\chi_{A} \bowtie \chi_{B} \bowtie \chi_{C}}\left(\varepsilon_{1} \gamma_{T_{1}}, \varepsilon_{2} \gamma_{T_{2}}, \varepsilon_{3} \gamma_{T_{3}}\right)=\frac{1}{2^{n}} \sum_{D \subseteq X_{n}}(-1)^{\left|A \cap\left(D \triangle T_{1}\right)\right|+\left|B \cap\left(D \triangle T_{2}\right)\right|+\left|C \cap\left(D \triangle T_{3}\right)\right|}
$$

for all $\varepsilon_{i}= \pm 1$ and $T_{i} \subseteq X_{n}, i=1,2,3$. In particular (when $T_{1}=T_{2}=T_{3}=\varnothing$ ), we have

$$
\psi_{\chi_{A} \bowtie \chi_{B} \bowtie \chi_{C}}(1,1,1)= \begin{cases}1 & \text { if } C=A \triangle B \\ 0 & \text { otherwise. }\end{cases}
$$


Indeed, in this case, (4.20) gives

$$
\begin{aligned}
\psi_{\chi_{A} \bowtie \chi_{B} \bowtie \chi_{C}}(1,1,1) & =\frac{1}{2^{n}} \sum_{D \subseteq X_{n}}(-1)^{|A \cap D|+|B \cap D|+|C \cap D|} \\
& =\frac{1}{2^{n}} \sum_{D \subseteq X_{n}}(-1)^{|(A \triangle B) \cap D|+2|A \cap B \cap D|+|C \cap D|} \\
& =\frac{1}{2^{n}} \sum_{D \subseteq X_{n}}(-1)^{|((A \triangle B) \triangle C) \cap D|+2|((A \triangle B) \cap C) \cap D|} \\
& =\frac{1}{2^{n}} \sum_{D \subseteq X_{n}}(-1)^{|((A \triangle B) \triangle C) \cap D|}
\end{aligned}
$$

and (4.21) follows from Lemma 4.2 by taking $U=(A \triangle B) \triangle C$.

Suppose now that $n$ is even. The spherical character on $\mathbb{C L}(n) \times \mathbb{C L}(n) \times \mathbb{C L}(n)$ associated with the representation $\rho_{n} \otimes \rho_{n} \otimes \rho_{n}$ is trivial. Indeed,

$$
\begin{aligned}
\psi_{\rho_{n} \boxplus \rho_{n} \boxplus \rho_{n}}\left(\varepsilon_{1} \gamma_{T_{1}},\right. & \left.\varepsilon_{2} \gamma_{T_{2}}, \varepsilon_{3} \gamma_{T_{3}}\right)=\frac{1}{2^{n+1}} \sum_{\varepsilon= \pm 1} \sum_{D \subseteq X_{n}} \prod_{i=1}^{3} \chi_{\rho_{n}}\left(\varepsilon \varepsilon_{i} \gamma_{D} \gamma_{T_{i}}\right) \\
& = \begin{cases}\frac{1}{2^{n+1}} \sum_{\varepsilon= \pm 1} \prod_{i=1}^{3} \chi_{\rho_{n}}\left(\varepsilon \varepsilon_{i}(-1)^{\xi\left(T_{i}, T_{i}\right)} \gamma_{\varnothing}\right) & \text { if } T_{1}=T_{2}=T_{3} \\
0 & \text { otherwise }\end{cases} \\
& = \begin{cases}\sum_{\varepsilon= \pm 1} \varepsilon\left(\frac{1}{2^{n+1}} \prod_{i=1}^{3}\left(\varepsilon_{i}(-1)^{\xi\left(T_{i}, T_{i}\right)} 2^{n / 2}\right)\right) & \text { if } T_{1}=T_{2}=T_{3} \\
0 & \text { otherwise }\end{cases} \\
& =0
\end{aligned}
$$

for all $\varepsilon_{i}= \pm 1$ and $T_{i} \subseteq X_{n}, i=1,2,3$.

The spherical character on $\mathbb{C L}(n) \times \mathbb{C L}(n) \times \mathbb{C L}(n)$ associated with the representation $\chi_{A} \otimes \rho_{n} \otimes \rho_{n}$ for $A \subseteq X_{n}$, is given by

$$
\begin{aligned}
\psi_{\chi_{A} \bowtie \rho_{n} \bowtie \rho_{n}}\left(\varepsilon_{1} \gamma_{T_{1}},\right. & \left.\varepsilon_{2} \gamma_{T_{2}}, \varepsilon_{3} \gamma_{T_{3}}\right)=\frac{1}{2^{n+1}} \sum_{\varepsilon= \pm 1} \sum_{D \subseteq X_{n}} \chi_{A}\left(\varepsilon \varepsilon_{1} \gamma_{D} \gamma_{T_{1}}\right) \prod_{i=2}^{3} \chi_{\rho_{n}}\left(\varepsilon \varepsilon_{i} \gamma_{D} \gamma_{T_{i}}\right) \\
& = \begin{cases}\frac{1}{2^{n+1}} \sum_{\varepsilon= \pm 1}(-1)^{\left|T \cap T_{1}\right|} \prod_{i=2}^{3}\left(\varepsilon \varepsilon_{i}(-1)^{\xi(T, T)} 2^{n / 2}\right) & \text { if } T_{2}=T_{3}=: T \\
0 & \text { otherwise }\end{cases} \\
& = \begin{cases}(-1)^{\left|T \cap T_{1}\right|} \varepsilon_{2} \varepsilon_{3} & \text { if } T_{2}=T_{3}=: T \\
0 & \text { otherwise }\end{cases}
\end{aligned}
$$

for all $\varepsilon_{i}= \pm 1$ and $T_{i} \subseteq X_{n}, i=1,2,3$. 
The spherical character on $\mathbb{C L}(n) \times \mathbb{C L}(n) \times \mathbb{C} \mathbb{L}(n)$ associated with the representation $\chi_{A} \otimes \chi_{B} \otimes \chi_{\rho_{n}}$, for $A, B \subseteq X_{n}$, is again trivial. Indeed,

$$
\begin{aligned}
\psi_{\chi_{A} \bowtie \chi_{B} \bowtie \rho_{n}}\left(\varepsilon_{1} \gamma_{T_{1}}, \varepsilon_{2} \gamma_{T_{2}}, \varepsilon_{3} \gamma_{T_{3}}\right) & =\frac{1}{2^{n+1}} \sum_{\varepsilon= \pm 1} \sum_{D \subseteq X_{n}} \chi_{A}\left(\varepsilon \varepsilon_{1} \gamma_{D} \gamma_{T_{1}}\right) \chi_{B}\left(\varepsilon \varepsilon_{2} \gamma_{D} \gamma_{T_{2}}\right) \chi_{\rho_{n}}\left(\varepsilon \varepsilon_{3} \gamma_{D} \gamma_{T_{3}}\right) \\
& =\frac{1}{2^{n+1}} \sum_{\varepsilon= \pm 1} \varepsilon\left(\chi_{A}\left(\gamma_{T_{3}} \gamma_{T_{1}}\right) \chi_{B}\left(\gamma_{T_{3}} \gamma_{T_{2}}\right)\left(\varepsilon_{3}(-1)^{\xi\left(T_{3}, T_{3}\right)} 2^{n / 2}\right)\right) \\
& =0
\end{aligned}
$$

for all $\varepsilon_{i}= \pm 1$ and $T_{i} \subseteq X_{n}, i=1,2,3$.

Suppose now that $n$ is odd. The spherical character on $\mathbb{C L}(n) \times \mathbb{C L}(n) \times \mathbb{C L}(n)$ associated with the representations $\rho_{n}^{\eta_{1}} \otimes \rho_{n}^{\eta_{2}} \otimes \rho_{n}^{\eta_{3}}$ is trivial for all $\eta_{i}= \pm, i=1,2,3$. Indeed,

$$
\begin{aligned}
& \psi_{\rho_{n}^{\eta_{1}} \bowtie \rho_{n}^{\eta_{2}} \bowtie \rho_{n}^{\eta_{3}}}\left(\varepsilon_{1} \gamma_{T_{1}}, \varepsilon_{2} \gamma_{T_{2}}, \varepsilon_{3} \gamma_{T_{3}}\right)=\frac{1}{2^{n+1}} \sum_{\varepsilon= \pm 1} \sum_{D \subseteq X_{n}} \prod_{i=1}^{3} \chi_{\rho_{n}^{\eta_{i}}}\left(\varepsilon \varepsilon_{i} \gamma_{D} \gamma_{T_{i}}\right) \\
& =\frac{1}{2^{n+1}} \sum_{\varepsilon= \pm 1} \varepsilon\left(\sum_{D \subseteq X_{n}} \prod_{i=1}^{3} \chi_{\rho_{n}^{\eta_{1}}}\left(\varepsilon_{i} \gamma_{D} \gamma_{T_{i}}\right)\right) \\
& =0
\end{aligned}
$$

for all $\varepsilon_{i}= \pm 1$ and $T_{i} \subseteq X_{n}, i=1,2,3$.

In order to express the spherical character on $\mathbb{C L}(n) \times \mathbb{C L}(n) \times \mathbb{C L}(n)$ associated with the representations $\chi_{A} \otimes \rho_{n}^{\eta_{2}} \otimes \rho_{n}^{\eta_{3}}$ for $A \subseteq X_{n}$ and $\eta_{i}= \pm, i=2,3$, let $\varepsilon_{i}= \pm 1$ and $T_{i} \subseteq X_{n}, i=1,2,3$. Then we have

$$
\begin{aligned}
\psi_{\chi_{A} \boxplus \rho_{n}^{\eta_{2}} \bowtie \rho_{n}^{\eta_{3}}}\left(\varepsilon_{1} \gamma_{T_{1}}, \varepsilon_{2} \gamma_{T_{2}}, \varepsilon_{3} \gamma_{T_{3}}\right) & =\frac{1}{2^{n+1}} \sum_{\varepsilon= \pm 1} \sum_{D \subseteq X_{n}} \chi_{A}\left(\varepsilon \varepsilon_{1} \gamma_{D} \gamma_{T_{1}}\right) \prod_{i=2}^{3} \chi_{\rho_{n}^{\eta_{i}}}\left(\varepsilon \varepsilon_{i} \gamma_{D} \gamma_{T_{i}}\right) \\
& =\frac{1}{2^{n}} \sum_{D \subseteq X_{n}} \chi_{A}\left(\gamma_{D \triangle T_{1}}\right) \prod_{i=2}^{3} \varepsilon_{i} \chi_{\rho_{n}^{\eta_{i}}}\left((-1)^{\xi\left(D, T_{i}\right)} \gamma_{D \triangle T_{i}}\right) .
\end{aligned}
$$

This quantity vanishes if $T_{2} \neq T_{3}, \overline{T_{3}}$ while, if $T_{2}=T_{3}:=T$ equals

$$
\begin{aligned}
\frac{1}{2^{n}}\left(\chi_{A}\left(\gamma_{T \triangle T_{1}}\right)\right. & \varepsilon_{2} \varepsilon_{3} \chi_{\rho_{n}^{\eta_{2}}}\left((-1)^{\xi(T, T)} \gamma_{T \triangle T}\right) \chi_{\rho_{n}^{\eta_{3}}}\left((-1)^{\xi(T, T)} \gamma_{T \triangle T}\right) \\
& \left.+\chi_{A}\left(\gamma_{\bar{T} \triangle T_{1}}\right) \varepsilon_{2} \varepsilon_{3} \chi_{\rho_{n}^{\eta_{2}}}\left((-1)^{\xi(\bar{T}, T)} \gamma_{\bar{T} \triangle T}\right) \chi_{\rho_{n}^{\eta_{3}}}\left((-1)^{\xi(\bar{T}, T)} \gamma_{\bar{T} \triangle T}\right)\right) \\
& =2^{n} \varepsilon_{2} \varepsilon_{3}\left((-1)^{\left|A \cap\left(T \triangle T_{1}\right)\right|}+c^{2} \eta_{2} \eta_{3}(-1)^{\left|A \cap\left(\bar{T} \triangle T_{1}\right)\right|}\right)
\end{aligned}
$$

and, finally, if $T_{2}=\overline{T_{3}}:=T$, equals

$$
\begin{aligned}
\frac{1}{2^{n}}\left(\chi_{A}\left(\gamma_{T \triangle T_{1}}\right)\right. & \varepsilon_{2} \varepsilon_{3} \chi_{\rho_{n}^{\eta_{2}}}\left((-1)^{\xi(T, T)} \gamma_{T \triangle T}\right) \chi_{\rho_{n}^{\eta_{3}}}\left((-1)^{\xi(T, \bar{T})} \gamma_{T \triangle \bar{T}}\right) \\
& \left.+\chi_{A}\left(\gamma_{\bar{T} \triangle T_{1}}\right) \varepsilon_{2} \varepsilon_{3} \chi_{\rho_{n}^{\eta_{2}}}\left((-1)^{\xi(\bar{T}, T)} \gamma_{\bar{T} \triangle T}\right) \chi_{\rho_{n}^{\eta_{3}}}\left((-1)^{\xi(\bar{T}, \bar{T})} \gamma_{\bar{T} \triangle \bar{T}}\right)\right) \\
& =2^{n} c \varepsilon_{2} \varepsilon_{3}\left(\eta_{3}(-1)^{\left|A \cap\left(T \triangle T_{1}\right)\right|+\xi(T, T)+\xi(T, \bar{T})}+\eta_{2}(-1)^{\left|A \cap\left(\bar{T} \triangle T_{1}\right)\right|+\xi(T, \bar{T})+\xi(\bar{T}, \bar{T})}\right) .
\end{aligned}
$$


As special cases (essentially the only ones for which we have a rather simple expression) we have

and

$$
\psi_{\chi_{A} \unrhd \rho_{n}^{\eta_{2}} \bowtie \rho_{n}^{\eta_{3}}}\left(\varepsilon_{1} \gamma_{T}, \varepsilon_{2} \gamma_{T}, \varepsilon_{3} \gamma_{T}\right)=2^{n} \varepsilon_{2} \varepsilon_{3}\left(1+(-1)^{|A|} \eta_{2} \eta_{3} c^{2}\right)
$$

$$
\psi_{\chi_{A} \unrhd \rho_{n}^{\eta_{2}} \boxplus \rho_{n}^{\eta_{3}}}\left(\varepsilon_{1} \gamma_{\bar{T}}, \varepsilon_{2} \gamma_{T}, \varepsilon_{3} \gamma_{T}\right)=2^{n} \varepsilon_{2} \varepsilon_{3}(-1)^{|A|}
$$

Finally, the spherical character on $\mathbb{C L}(n) \times \mathbb{C L}(n) \times \mathbb{C L}(n)$ associated with the representations $\chi_{A} \otimes \chi_{B} \otimes \rho_{n}^{ \pm}$is again trivial for all $A, B \subseteq X_{n}$. Indeed,

$$
\begin{aligned}
\psi_{\chi_{A} \bowtie \chi_{B} \bowtie \rho_{n}^{ \pm}}\left(\varepsilon_{1} \gamma_{T_{1}}, \varepsilon_{2} \gamma_{T_{2}}, \varepsilon_{3} \gamma_{T_{3}}\right) & =\frac{1}{2^{n+1}} \sum_{\varepsilon= \pm 1} \sum_{D \subseteq X_{n}} \chi_{A}\left(\varepsilon \varepsilon_{1} \gamma_{D} \gamma_{T_{1}}\right) \chi_{B}\left(\varepsilon \varepsilon_{2} \gamma_{D} \gamma_{T_{2}}\right) \chi_{\rho_{n}^{ \pm}}\left(\varepsilon \varepsilon_{3} \gamma_{D} \gamma_{T_{3}}\right) \\
& =\frac{1}{2^{n+1}} \sum_{\varepsilon= \pm 1} \varepsilon\left(\sum_{D \subseteq X_{n}} \chi_{A}\left(\gamma_{D} \gamma_{T_{1}}\right) \chi_{B}\left(\gamma_{D} \gamma_{T_{2}}\right) \chi_{\rho_{n}^{ \pm}}\left(\varepsilon_{3} \gamma_{D} \gamma_{T_{3}}\right)\right) \\
& =0
\end{aligned}
$$

for all $\varepsilon_{i}= \pm 1$ and $T_{i} \subseteq X_{n}, i=1,2,3$.

\section{REFERENCES}

[1] T. Ceccherini-Silberstein, F. Scarabotti and F. Tolli, Finite Gelfand pairs and their applications to probability and statistics, J. Math. Sci. (N. Y.) 141 (2007), no. 2, 1182-1229.

[2] T. Ceccherini-Silberstein, F. Scarabotti and F. Tolli, Harmonic analysis on finite groups: representation theory, Gelfand pairs and Markov chains. Cambridge Studies in Advanced Mathematics 108, Cambridge University Press 2008.

[3] T. Ceccherini-Silberstein, F.Scarabotti and F.Tolli: Representation Theory and Harmonic Analysis of wreath products of finite groups. London Mathematical Society Lecture Note Series 410, Cambridge University Press, 2014.

[4] T. Ceccherini-Silberstein, F. Scarabotti and F. Tolli, Mackey's theory of $\tau$-conjugate representations for finite groups, arXiv:1311.7252,

[5] P. Diaconis, Groups Representations in Probability and Statistics. IMS Hayward, CA, 1988.

[6] F. Scarabotti and F. Tolli, Harmonic analysis on a finite homogeneous space, Proc. Lond. Math. Soc.(3) 100 (2010), no. 2, 348-376.

[7] B. Simon, Representations of finite and compact groups, American Math. Soc., 1996.

Dipartimento di Ingegneria, Università del Sannio, C.so Garibaldi 107, 82100 BenEVENTO, ITALY

E-mail address: tceccher@mat.uniroma1.it

Dipartimento SBai, Sapienza Università di Roma, via A. Scarpa 8, 00161 Roma, Italy

E-mail address: fabio.scarabotti@sbai.uniroma1.it

Dipartimento di Matematica e Fisica, Università Roma Tre, L. San Leonardo Murialdo 1, 00146 Roma, Italy

E-mail address: tolli@mat.uniroma3.it 